\title{
TEORIA Y POIESIS EN TOMAS HOBBES
}

\section{Diana María López}

"Observé que de la posesión común de las cosas debe surgir necesariamente la guerra y, con ella, todas las formas de la misma para los hombres, los cuales se enemistan violentamente por su disfrute, a pesar de que por naturaleza todos aborrecen este estado. De este modo, fui a parar a las dos exigencias más seguras de la naturaleza humana: la una es la exigencia de los deseos naturales, por mor de la cual uno reclama para sí solo el uso de las cosas comunes; la otra es la exigencia de la razón natural, por mor de la cual cada uno busca evitar la muerte violenta en tanto que máximo mal de la naturaleza. A partir de estos fundamentos creo haber expuesto la necesidad de contratos y del cumplimiento de la palabra dada, y con ello, los elementos de las virtudes morales y de los deberes ciudadanos... en clara argumentación":

T. Hobbes: Elementos de Derecho Natural y Polftico.

\section{Introducción}

Desde Descartes la cuestión central de la filosofía moderna ha sido la de cómo es posible un conocimiento seguro.

El propósito de elaborar una ética, una ciencia del derecho, una política (a la que se agrega también al final una economía), en suma una filosofía práctica demostrativa, es decir, apoyada en principios evidentes y deducida de estos principios de manera lógicamente rigurosa, constituye el ambicioso proyecto de Tomas Hobbes. El programa de Bacon: scientia propter potentiam, saber es poder, desborda el ámbito de las ciencias naturales cuando Hobbes declara a la conducta humana objeto legítimo de conocimiento científico, y emprende una construcción de la ciencia de la política capaz de conectar definitivamente: la naturaleza del hombre, el "estado natural" de la sociedad y el ideal del orden jurídico y estatal. 
La clásica división entre teoría y praxis, y la consiguiente distinción entre ciencias teóricas y ciencias prácticas, sufre una drástica alteración a la luz del moderno concepto de ciencia, el cual se construye sobre la base de dos pilares fundamentales: la combinación de la matemática con el experimento y la concepción del mundo físico según la cual, cada miembro se halla enlazado con otro causalmente según leyes mecánicas. El espíritu científico del siglo XVII, inclinado a demostrar la verdad more geométrico y a sostener el ideal de un conocimiento seguro, que dé cuenta de la totalidad de lo real partiendo de principios evidentes, condiciona profundamente la comprensión de la naturaleza humana, y, en consecuencia, la justificación racional de las condiciones más adecuadas para la ordenación de la vida en sociedad.

En una cosmovisión desde la cual, el orden social y político comparte el mismo modelo causal y estructural que la naturaleza; en la cual, el análisis descompone igualmente realidades físicas en partes, lo mismo que la sociedad en unidades atómicas; en la que, el poder político apartado de toda trascendencia tiende a cuantificarse hasta convertirse en organización técnica, y, a partir de la cual, el movimiento deductivo de la razón infiere de un tan hipotético como a-histórico "estado de naturaleza", la formación del orden social, nos preguntamos: ¿puede ser considerada "ciencia" una comprensión prudente de situaciones cambiantes con vistas a lo que debe hacerse? ¿Pueden la formación del carácter virtuoso, la vida buena y justa, y el cultivo de la prudencia práctica, ser objeto de estudio exacto y apodíctico cuando se parte de la premisa según la cual lo que define la condición del hombre no es la pertenencia a una comunidad y a su pasado, sino las determinaciones pre-políticas de una abstracta "naturaleza humana"?.

El camino inaugrado hacia un análisis gnoseológico más exacto de la 'praxis', daba lugar a dos posibilidades en apariencia excluyentes: eliminar a la política del ámbito de las consideraciones científicas o elaborar este ámbito sobre la imagen y modelo de las ciencias teóricas. La opción por la última ha conducido, y no con pocas resistencias (1),

(1) MONTAIGNE, M.: Ensayos: "Verdaderamente tengo curiosidad cuando, para dar alguna certidumbre a las leyes, sostienen que hay algunas estables, 
a la disolución de la filosofía práctica en sentido clásico, a la cientifización de la política y a la pérdida de un concepto más comprensivo de razón en favor de la validéz exclusiva del pensamiento científico. T. Mc Carthy, siguiendo a Habermas, pone de manifiesto de qué manera la absorción de la esfera de lo práctico por la esfera de lo técnico, encuentra aquí el origen de su desplazamiento: " . . el problema práctico de la vida virtuosa de los ciudadanos de la polis, se transformó en el problema técnico de cómo regular la interacción social para asegurar el orden y bienestar de los ciudadanos del Estado". (2)

perpetuas e inmutables" (. . .) "de las tres o cuatro seleccionadas no hay una sola que no sea contradicha y desmentida no sólo por un pueblo sino por muchos" (cit. por Bobbio, N.: Sociedad y Estado en la Filosofia Moderna, F.C.E. pág. 21);

VICO, G.B.: La Scienza Nuova Prima: "Lo que se siente justo por todos o la mayor parte de los hombres, debe ser la regla de la vida social". . . "cualquiera que quiera sobrepasar estos límites que deben ser las fronteras de la razón humana, debe cuidar de no sobrepasar toda la humanidad " (cit. por Bobbio, N.: pág. 42-43);

VICO, G.B.: "puesto que la finalidad de los estudios es la verdad, dirigmos nuestra investigación a la naturaleza de las cosas, porque ella parece ser cierta; pero no investigamos la naturaleza del hombre, porque ella es completamente incierta a causa del libre arbitrio" (cit. por Hennis, W. en Política y Filosofía Práctica, pág. 64-65);

MEINECKE, F.: "Toda la tarea del historicismo ha consistido en debilitar y hacer frágil el rígido pensamiento iusnaturalista, con su fe en la invariabilidad de la naturaleza humana". En El historicismo y su génesis, F.C.E., México, 1943

CORCE, B.: en su referencia a Rosseau, condena las "construcciones geométricas y mecánicas" de toda la escuela del dereco natural, creada cuando se desarrollaba y crecía la ciencia matemática de la naturaleza, y del hábito mental, a la filosofía, a la historia, a la política ". En Etica y Política, Laerza, p. 257

(2) MC CARTHY, T.: La teoría crítica de J. Habermas, Madrid Tecnos, 1987, pág. 22 
La ciencia no es ya conocimiento de cosas sino de causas: no hay más ciencia que de lo demostrable a priori. Así la geometría es ciencia porque se ocupa de figuras que nosostros mismos construímos. Si es verdad que la geometría es "la única ciencia que hasta ahora le haya gustado a Dios para regalársela al género humano", la única ciencia "cuyas conclusiones ahora ya se han vuelto indiscutibles", al filósofo moral le compete imitarla: "Puesto que las causas de las propiedades que tienen las figuras particulares residen en las líneas que nosotros mismos trazamos, y puesto que la producción de figuras depende de nuestra voluntad, por ésto, para el conocimiento de cualquier propiedad de una figura no es exigible otra cosa que extraigamos todas las consecuencias de la construcción que nosotros mismos llevamos a cabo en el dibujo de la figura. Por este motivo, porque nosotros mismos producimos las figuras, la geometría es tenida por una ciencia demostrativa. . . Además de ésto, cabe demostrar de una forma a priori la política y la ética, ésto es, la ciencia de la justicia y de la injusticia, de la equidad y la iniquidad, porque nosotros mismos creamos los principios para el conocimiento de la esencia de la justicia y de la equidad. ..., esto es, las causas de la justicia, a saber: las leyes y convenios" (De Cive, II, pp. 17 ss.).

Precisamente, a causa de la falta de un método riguroso, y por ende, de un parámetro único desde el cual distinguir lo verdadero de lo falso, los conocimientos que resultan de la interpretación y explicación de los fenómenos en el ámbito de la acción, se reducen a una sucesión de opiniones que contradiciéndose, coexisten sin excluirse. Una renovación de los estudios sobre la conducta humana no puede pasar sino a través de un cambio de método, el cual debe garantizar: certeza apodíctica, abstracción histórica y neutralidad axiológica.

Para Hobbes, quien ve a la ciencia del comportamiento humano formando parte de la mecánica universal, los vínculos entre el pensamiento científico y el pensamiento político, resultan evidentes: "Ya a propósito de un reloj que se mueve a sí mismo y a propósito de toda máquina algo complicada (en analogía con la cual se interpretaba por aquel entonces la naturaleza en su totalidad), no puede comprenderse la actitud de las partes y engranajes particulares, si no se toman separadamente y se considera por sí la materia, la forma y el movimiento de cada parte. De igual modo, a propósito de los derechos del Estado y de los 
deberes del ciudadano, debe investigarse la naturaleza humana, debe investigarse en qué medida es adecuada, o no para la formación del Estado, y cómo tienen los hombres que aliarse si es que desean convertirse en una unidad" (De Cive, VI).

En este sentido, descubrir las reglas universales de la conducta por medio del estudio de la naturaleza del hombre, será el punto de partida de la construcción de una ciencia moral capaz de dar cuenta de la conformidad o disconformidad de las acciones humanas a esas reglas, las cuales pueden ser conocidas con seguridad cuando se abandone el terreno de los hechos singulares y se comiencen a considerar las causas universales y necesarias que las producen. En el ámbito de la política, el cuerpo político (the artificial body) imaginado como un sistema de pesos, contrapesos, balanzas y frenos, es producido lógicamente de manera deductiva y justificado, no por el estudio histórico de la evolución de la sociedad, sino por razonamientos formulados demostrativamente.

Según Bobbio, "la doctrina iusnatural ista del Estado -que encuentra en Hobbes uno de sus principales inspiradores-, no es solmente una teoría racional del Estado sino también una teoría del Estado racional. Esto quiere decir que desemboca en una teoría de la racionalidad del Estado en cuanto construye el Estado como ente de razón por excelencia, en el que solamente el hombre realiza plenamente su naturaleza de ser racional. Si es verdad que para el hombre en cuanto criatura divina extra eclesiam nulla salus, es igualmente verdad que para el hombre en cuanto ser natural y racional no hay salvación extra republicam. (3)

"Fuera del Estado -dice Hobbes en De Cive, X, 1- es el dominio de las pasiones, la guerra, el miedo, la pobreza, el abandono, el aislamiento, la barbarie, la ignorancia, la bestialidad. En el Estado es el dominio de la razón, la paz, la seguridad, la riqueza, la decencia, la sociabilidad, el refinamiento, la ciencia, la benevolencia".

La construcción de una ética racional, separada definitivamente de la teología y capaz por sí misma de garantizar la universalidad de los

(3) BOBBIO, N.: Op. cit.: pág. 129. 
principios de la conducta humana; de una doctrina juridica, según la cual las leyes civiles no son otra cosa que las mismas leyes naturales, dotadas de poder coactivo para obligar a los hombres a coexistir en un marco de racionalidad; de una teoría poltítica que eleva al Estado no solamente a sede de la vida racional, sino en definitiva, a la única salida para poner fin a la guerra de todos contra todos y garantizar la seguridad del ciudadano, se configura como: producto del contexto social, es decir, del proceso de secularización de la autoridad y, en general, de la vida civil; resultado del progreso científico, que revoluciona las nociones y las matrices de pensamiento; y como respuesta a un hecho extremadamente humano, las pasiones, consideradas más tarde por Spinoza en su Tratado Político: "...no como vicios sino como propiedad de la naturaleza humana, de la misma manera que a la naturaleza de la atmósfera le corresponden el calor, el frío, la tempestad, el rayo y cosas semejantes". (4)

Hobbes funda sobre estas bases, su ideal de un orden moral, jurídico y estatal, en el cual la unidad de la voluntad del soberano, mantiene en la paz a los sujetos individuales y a la sociedad que se compone de ellos.

Esta filosofía se coloca en las antípodas de la filosofía práctica en sentido clásico, no sólo por su método que intenta reducir el derecho, la moral y la política por primera vez en la historia de la reflexión sobre la conducta humana a ciencia demostrativa; no sólo por su teoría del poder, la cual legitima la laicización del Estado y la primacía de la ley sobre la costumbre, sino y fundamentalmente, por su concepción de la razón, la cual según Pufendorf ". . aún en el estado de naturaleza posee un criterio de evaluación común, seguro y constante. . .", pero que en realidad, no es más que un instrumento al servicio del cálculo para justificar el Estado civil absoluto sobre la base del pacto, de manera tal que como dice Spinoza ". . .cada uno transfiera todo su poder a la

(4) En Sociedad y Estado en la filosofía moderna, Bobbio expresa: ". . .con Locke y Kant, los intereses tomarán el lugar de las pasiones como clave de la vida social, pero la antítesis "interés individual-interés social", jamás eliminará del todo la antítesis 'pasión-razón' ". 
sociedad, la cual detentará de esta forma y por sí sola, el derecho natural superior a todo, es decir, el supremo poder al que cada uno debería obedecer".

Como han señalado diversos autores, Hobbes representa un momento clave en la evolución de concepciones no sólo diferentes sino en extremo divergentes. Para Ferdinand Tönnies, su pensamiento pone de manifiesto la transición del absolutismo monárquico al liberalismo. Leo Strauss, lo reconoce como un autor que expresa la evolución de la moral de las virtudes aristocráticas (honor, gloria) a las virtudes burguesas inspiradas en el temor, la previsión y la prudencia individualista. Para Dilthey, por su parte, representa el eslabón de enlace entre la escuela atomista de la Antigüedad, la física mecánica, su concepción de la naturaleza del hombre, su derecho natural racional y el materialismo y el positivismo de los siglos XVIII y XIX. Coincidimos, finalmente, con autores como Bobbio y Touchart para quienes con Hobbes, no sólo nos encontramos frente a una nueva doctrina jurídica o a una original teoría del poder, sino ante la expresión de un movimiento cultural, filosófico, científico, religioso y político, cuyos efectos de sentido nos son hoy contemporáneos y cuyos objetivos fundacionales orientaron el pensamiento y la acción, en la línea de la sustitución definitiva de la virtud por el bienestar, de la justicia por la paz, de la felicidad por la libertad.

\section{La transición}

A fin de tematizar con mayor profundidad la ruptura de la filosofía hobbesiana con relación a la tradición, así como los ejes fundamentales de su pensamiento en relación con la construcción de una teoría de la moral y del poder con base científica, consideramos pertinente revisar este proceso tomando como punto de partida la fuente determinante de la tradición: Aristóteles.

Para Aristóteles, lo que explica la diferencia entre teoría y praxis, es un contenido objetivo central: los conocimientos teóricos se buscan por sí mismos, los prácticos por causa del actuar justo. El ámbito de la praxis, es decir de la vida activa, es el ámbito de la elección consciente. Los objetos de las ciencias teóricas tienen su ser sin la acción de los 
hombres. Las ciencias de las acciones de los hombres, encuentran el principio de esta acción, no en el objeto al cual se dirige, sino en el que actúa. Por el contrario, la ciencia teórica se ocupa de objetos que tienen y llevan en sí mismos el principio de su movimiento. Aquí es posible un conocimiento puramente teórico. Aquí trata el científico de entender puramente lo que es. Modificar los objetos del conocimiento científico, es algo para lo cual él no está capacitado ni algo que habrá de desear.

Diferente es el propósito cognoscitivo en el ámbito de las cosas humanas. Aristóteles insiste en que la reflexión tiene lugar aquí, solamente a consecuencia y bajo el aspecto de querer influir sobre el propio comportamiento con vistas a su perfección. Aquí no se trata simplemente de comprender -al modo de una comprensión libre de juicio valorativo- sino de la comprensión que está en situación de servir a la conducción de la vida. El "fin aquí no es el conocimiento puro, sino la acción". (5)

La causa más importante de la diferenciación del ámbito de la praxis de la esfera del conocimiento teórico, es para Aristóteles en consecuencia, el carácter no-necesario de sus objetos. En el ámbito de la teoría no hay ninguna probabilidad, alli reina una rigurosa necesidad. De manera diferente acontece en el campo de la praxis, en donde el principio se encuentra en el actor. Aqur la realidad -la vida comunitaria, las instituciones políticas- puede ser racional pero no necesita serlo. El comportamiento humano, el obrar, el campo de la política, no están determinados. Cada acción es la realización de una posibilidad. Cuál entre las diversas posibilidades es aquella que se escoge, depende de una selección que no tiene lugar sobre la base de leyes necesarias. Aquello a lo que puede tenderse, en el campo de praxis, es el conocimiento de probabilidades que determinan la dirección de las acciones. Las premisas y las consecuencias en el ámbito de las acciones prácticas, expresan por tanto, verdades generales pero no apodícticas.

El hecho de que las ciencias prácticas no se ejerzan por sí y para sí mismas, sino que la investigación en este ámbito esté orientado al

(5) ARISTOTELES: Etica Nic., I. 1, 109 a 5-6; 2, 1103 b 27. 
cultivo del carácter con vistas a llevar una vida buena y justa, determinaba su status cognitivo frente a las ciencias teóricas: lo que se ejerce por sí y para sí meramente con el fin del conocimiento, se considera en grado más alto como ciencia que lo que es recomendable por su utilidad. (Met. A, 2, 982 a).

En esta diferencia de grado de la filosofía práctica frente a la episteme en tanto ciencia rigurosa y, por ende, al impedimento de alcanzar en este campo un conocimiento apodíctico propio de un saber ahsoluto, radicaría el punto de contacto con la concepción moderna, segin la cual sólo deben considerarse "científicas" aquellas formas de saher que reposan en un "conocimiento cierto y evidente". En la 2 da. de las Reglas para la dirección del espíritu, Descartes afirma: ". . .debemos ocuparnos únicamente de aquellos objetos que nuestro espíritu parece poder conocer de un modo cierto e indudable".

El criterio de precisión y de exactitud exigido por Aristóteles para la episteme, pareciera avalar el abandono de la praxis de la esfera de las consideraciones científicas operado en el pensamiento moderno. Sin embargo, debemos destacar una diferencia fundamental: para Aristóteles, la exactitud del geómetra es una exactitud diferente a la del político. En el conocimiento de lo justo y de lo injusto no es posible alcanzar la misma certeza que logra el razonamiento matemático y resulta necesario conformarse con un conocimiento probable, justamente porque ". . Sería igualmente inconveniente -señala en la Etica Nicomaquea 1094 b- exigir demostraciones de un orador, que contentarse con la probabilidad en los razonamientos de un matemático".

Descartes no reconoce esta diferencia: la preocupación por el método, la obsesión por la exactitud, la autoridad indiscutible otorgada a la Aritmética y a la Geometría en tanto paradigmas desde los cuales distinguir lo que es saber de lo que no lo es, le impiden admitir conocimientos que por regla general, sólo son probablemente válidos: "Rechazamos -dice en la Regla II-todos los conocimientos meramente probables y establecemos que no se debe creer sino en los perfectamente conocidos y respecto de los cuales no se puede dudar".

(6) DESCARTES, R.: Obras Escogidas, Buenos Aires, Charcas, 1980, pág. 37-38. 
En el pensamiento de Aristóteles, teoría y praxis se distinguen, pero el hecho de que la primera se construya sobre la base de afirmaciones evidentes $y$, la segunda, contemple juicios de valor susceptibles de variación conforme a situaciones cambiantes, no disuelve a la filosofía práctica en la esfera de la episteme ni la relega al lugar de lo incomunicable; se trata, en definitiva, de dos ámbitos de la actividad humana distintos o irreductibles. En cambio en Descartes, su visión unilateral del conocimiento, le obliga a subordinar la filosofía práctica a las exigencias de un concepto de ciencia puramente teórica: en su obra sobre Las pasiones del alma, la Etica es puesta en conexión con el nuevo ideal de ciencia al expresar que él ha querido tratar sobre las pasiones ". . .no como orador, ni como moralista, sino como conocedor de la naturaleza". Lo que hasta entonces sẹ había llamado 'vicio' y 'virtud', se trata ahora de comprender como el producto de determinadas causalidades. Se deben investigar las "causas primeras" de las pasiones. "Las más habituales y las principales" de éstas son los objetos sensibles que las despiertan. El conocimiento del alma, del comportamiento humano, se ha convertido en un problema no de la vida activa sino de la actividad teórico-especulativa: el objetivo formulado en las Meditaciones de ". . .estabilizar al fin algo firme y permanente en las ciencias", supone -como él mismo lo expresa- apartarse de todo lo que "se refiere a la fe o a la conducción de la vida".

Como lo expresáramos en la introducción a este trabajo, Hobbes emprende la tarea de estudiar las acciones de los hombres y sus consecuencias prácticas, sobre la base de la transformación del ideal de ciencia formulada por sus contemporáneos. Valga el texto de su obra Elementos de Derecho Natural y Politico, que transcribimos a continuación, a fin de visualizar no sólo las afinidades con el pensamiento cartesiano, sino además, su opción por la "infalibilidad de la razon", la cual determinará no sólo el modelo de ciencia sino también la orientación definitiva de la elaboración de la totalidad de su sistema: "De las dos partes principales de nuestra naturaleza, Razón y Pasión, proceden las dos clases de conocimiento: matemático y dogmático. El primero está libre de disputas y controversias, porque consiste únicamente en comparar cifras y movimientos, cosas en que la verdad y el 
interés de los hombres no se oponen entre sí. Pero en el segundo no hay nada que no se pueda discutir, porque compara a los hombres y trata de sus derechos y beneficios; de forma que tantas veces como esté la razón en contra del hombre, otras tantas estará el hombre en contra de la razón. De lo que se desprende que todo lo que han escrito los hombres acerca de la justicia y la política en general se halla plagado de contradicciones de unos con otros y consigo mismos. Para reducir esta doctrina a las reglas y a la infalibilidad de la razón no existe otro medio que establecer unos principios básicos tales que, al no inspirar desconfianza a la razón, no se intente desplazarlos; y a continuación fundamentar gradualmente a partir de ellos, la verdad de las cosas en la ley natural (que hasta ahora ha sido establecida en el aire) de modo que el conjunto resulte inexpugnable."

Estudiar el mundo de la vida activa con los mismos instrumentos conceptuales con que los físicos estudian el mundo natural y, prescindiendo de todo aquello que pueden haber dicho los anteriores autores, optar por la vía de una reconstrucción racional del origen y fundamento de la sociedad y del Estado, serán los ejes de este proyecto.

Según Bobbio: "Antes de Hobbes, los tratados de filosofía política se apoyaban monótonamente sobre dos pilares, de tal manera que aparecían frecuentemente, sin más, como una repetición de lo que ya se había dicho: la Política de Aristóteles y el derecho romano" . . ."Junto con la autoridad de la historia -continúa Bobbio- Hobbes suprime la autoridad de Aristóteles, contra el que toma posición desde las primeras páginas del De Cive, contraponiendo a la hipótesis del hombre "naturaliter socialis", la hipótesis del "homo homini lupus". Hace tabla rasa de todas las opiniones precedentes y construye su teoría sobre bases sólidas, indestructibles del estudio de la naturaleza humana, además de la única manera posible dados estos supuestos, de satisfacerlas". (7)

En el desarrollo de su pensamiento, que sistemáticamente se organiza sobre la trilogía De Corpore (Tratado del Cuerpo), De Homine

(7) BOBBIO, N.: Op. Cit.: págs. 50-51 
(Tratado del Hombre) y De Cive (Tratado del Ciudadano), para culminar en Leviathán, síntesis de su obra, se manifiesta la intencionalidad no sólo de separarse de la tradición de una filosofía práctica que fue para él "más un sueño que una ciencia", sino además, de sentar las bases de un conocimiento capaz de dar cuenta de las relaciones de las acciones humanas, con la misma exactitud con la que se conocen las relaciones de dimensiones de las figuras geométricas.

La formulación del problema sobre un orden social justo, debe ser planteado de tal manera que permita un tratamiento unívoco, es decir, absolutamente exacto: ningún conocimiento debe ser confuso, probable o dudoso; en la esfera de lo rigurosamente demostrable la verdad se impone por sí sola. En este orden, la seguridad de que la diversidad de hechos singulares se explica desde causás universales y simples, justifica la puesta en marcha de un programa según el cual, la filosofia moral y política podrían ganar cientificamente mediante su reducción a ciencia demostrativa.

Resolveriamos en un juicio apresurado el pensamiento que nos ocupa, si lo juzgáramos solamente como "la nueva concepción del materialismo mecanicista y del derecho natural fundado en el egoísmo". La significación histórica de la obra de Hobbes, lugar de encuentro de pensadores de la Antigüedad (Demócrito, Tucídides, Epicuro) y de una tradición de la cual se distancia polémicamente; así como, punto de partida de corrientes de pensamiento cuyos efectos de sentido se aprecian en posiciones actuales, se distingue particularmente por su noción de "filosofía" comprendida como "ciencia universal", la cual orienta la construcción de la totalidad de su sistema: "Pregunto cuántas ciencias hay. Existe una sola ciencia universal que denominamos filosofia". Para continuar diciendo: "la filosoffa es el conocimiento de los fenómenos o efectos, a partir del conocimiento de sus posibles causas o de los fundamentos que los producen, y al revés, de los posibles fundamentos que los producen a partir de los efectos conocidos" (De Corpore I, 1, cap. 2) Hobbes distingue, el conocimiento de los efectos a partir de las causas productoras -el conocimiento demostrativo a priori que sólo es posible sobre cosas que pueden ser llevadas a cabo por el mismo hombre-, del conocimiento de las causas productoras a partir de 
los efectos conocidos, así pues, el conocimiento demostrativo a posteriori al que estamos abocados a propósito de cosas naturales cuya producción no está en nuestro poder. Ejemplos son, de un lado la geometría, de otro, la física. La filosofía social, en este orden, debe corresponder a la geometría en el hecho de que tiene que ver con relaciones (contratos) que producen los mismos hombres; por otra parte, es ciencia de la experiencia como la física, pues "... para conocer las propiedades del Estado es necesario que se conozcan previamente las predisposiciones, afectos y costumbres de los hombres". (De Cive I, 13).

Dilthey, en su análisis del pensamiento de Hobbes, destaca particularmente este aspecto ":... este tipo de visión del mundo surge cuando la filosofía toma su punto de partida en el estudio del mundo exterior. Justamente es en la ciencia natural, donde se realiza de la manera más cabal el concepto de un conocimiento de la conexión causal según leyes. Las investigaciones positivas se convierten en filosofía porque entran en la conexión de una deducción a partir de las verdades más generales que hacen referencia a todos los dominios del conocimiento" (...). "La unidad de todos los conocimientos como filosofía se limita, en el sentido de las ciencias positivas, a un conocimiento causal que subordina los hechos espirituales a los hechos físicos, es decir, a la inmensa conexión física del universo, y de las relaciones causales que se dan en la sociedad, los sentimientos morales, las instituciones jurf́dicas y la estructura de la vida política"... "La natruraleza del hombre - concluye Dilthey - se estudia en la sociedad pero marchando retrospectivamente desde el orden jurídico-político a sus causas, las cuales se hallan en la naturaleza humana" (8).

En este sentido, las condiciones del orden jurídico, moral y político radican en la naturaleza misma del hombre, de la cual se infiere por una cadena de deducciones, el sistema total de la vida social. Así resulta que: de la necesidad de suprimir el 'estado de naturaleza' se deduce el interés común por la paz y el orden; de la necesidad de hacer

(8) DILTHEY, W.: Hombre y Mundo en los siglos XVI y XVII, México, F.C.E., 1978, pág. 389, 390, 392. 
posible una supervivencia en paz y en orden se deduce el sistema contractual; de la necesidad de conservar el acuerdo del cual resulta el sistema contractual; se deduce el carácter soberano y absoluto del poder.

El 'estado civil', la sociedad que resulta del pacto, no puede ser otra cosa más que lo que es, pues se justifica en su racionalidad a partir de las leyes de la naturaleza misma: "Pues cada uno desea aquello que es bueno para él -dice Hobbes- y rehuye aquello que es malo; rehuye sobre todo el mayor de los de los males naturales, la muerte; y ciertamente a causa de una necesidad natural no menos que aquella en virtud de la cual una piedra cae hacia la tierra". La "legalidad" de las acciones humanas, semejante a la legalidad que rige la regularidad de los fenómenos naturales, constituye el supuesto previo para toda ampliación del conocimiento en el campo del espíritu y de la sociedad. Dada la importancia que adquiere esta noción en el sistema hobbesiano, no sólo por su carácter apriorístico, sino fundamentalmente, porque sobre él giraría el núcleo de la diferencia con la tradición, proponemos considerar su eficacia conceptual en la totalidad del marco teórico del autor.

Sabido es que en el campo de las ciencias naturales, la necesidad y el determinismo que caracterizan a la relación entre los fenómenos, posibilita no sólo la predicción sino además, la producción de estados futuros: un efecto puede ser provocado o evitado conociendo la causa que lo produce. Llevado este modo de explicar y manipular procesos objetivados a la esfera de las acciones humanas, supone entender la conducta de los otros en términos de regularidades observables y, por tanto, efectivamente controlables. Sobre esta base, el descubrimiento de la legalidad que rige el comportamiento humano, no sólo posibilitaría "describir". los fenómenos sociales, sino construir a priori las condiciones más adecuadas para la ordenación de la vida social.

He aquí justamente el paso fundamental dado por Hobbes con respecto a la tradición. Wilhelm Hennis en su obra Política y Filosofia Práctica afirma: "Lo especial, que hizo época, de la filosofía política de Hobbes radica en la combinación de un ideal teórico de conocimientos con presupuestos poiéticos. Los problemas de la filosofía política no 
se refieren ya a la vida justa, a la praxis, sino a una obra, una producción; es en su núcleo poiética" (9)

La profundización de este aspecto, al que consideramos muy importante en función del tema que nos ocupa, nos exige contrastarlo nuevamente con la doctrina clásica. Para Aristóteles, el conocimiento productivo no sólo se distingue del conocimiento teórico -en cuanto este último se ocupaba de un orden de realidad que no podía ser objeto de producción ni de acción-, sino también del conocimiento práctico. El ámbito de la praxis, o de la acción político-moral, quedaba reservado a la 'prudencia práctica', en tanto que el de la poiesis, o de la producción de útiles, pertenecía propiamente a la habilidad artesanal o techne.

En Hobbes, têrminos como "producción", "construcción", "habilidad", . . .se resignifican a la luz de nuevos intereses: ya no se trata de cómo "debe" vivir el hombre sino, bajo qué condiciones "puede" vivir tal como él "quiere" vivir, es decir, de poder establecer un estado capaz de hacer posible la vida deseada por todos. Para la producción de este estado artificial, no importa tanto como viven los hombres su praxis, y menos aún como deben vivir, sino que todo depende de la correcta construcción, de la manera exacta de crear este 'cuerpo artificial' (artificial body). "Si por causas de perturbaciones internas -dice Hobbes- Ilegase a disolverse un Estado, el defecto no descansaría en los hombres en cuanto son la materia de un Estado, sino en cuanto son los hacedores, los constructores del mismo" (De Corpore, I, Cap. $1 * 6)$.

El problema no es un problema de la praxis, de la justa y correcta conducción de la vida, sino del "producir", de la poiesis. A la frase tan frecuentemente citada: scientia propter potentiam la ciencia sirve solamente al poder, sigue la no menos significativa: "La teoría sirve solamente a la construcción".

La teoría se legitima en la práctica: el esfuerzo del conocimiento está justificado si conlleva la respuesta satisfactoria a necesidades y

(9) HENNIS, W.: Política y Filosofia Práctica, Bs. As., Sur, p. 56. 
deseos humanos. Así como el geómetra, utiliza los conocimientos adquiridos en la construcción de figuras para medir el campo y las aguas, y el saber del astrónomo lo coloca en condiciones de determinar el tiempo, la finalidad del saber no es un obrar correcto sino un "hacer": dadas las disposiciones del hombre que derivan de su naturaleza psicológica, dadas las relaciones en que la naturaleza ha puesto a los individuos entre sí (estado de naturaleza), y estableciendo el fin al que la misma naturaleza dirige la actividad humana, se trata de deducir el sistema de las condiciones más adecuadas para la realización de este fin (estado civil). Se trata, en definitiva, de mostrar el proceso a través del cual se pasa, deductivamente y por necesidad causal, al estado civil a partir del supuesto lógico del estado de naturaleza (De Cive, Prefacio; Leviatán I, c 13).

El rechazo de la problemática moral según lo formulara la fillosofía práctica, el desplazamiento del acento de lo práctico-dialéctico hacia lo poiético-teórico, se ha cumplido. "Hobbes traza la raya final debajo de la filosofía práctica tradicional -señala Hennis- cuando considera demostrado que la "prudencia" no es parte alguna de la filosofía, el alcance de esta separación de la sabiduría práctica (phronesis) del campo de la filosofía se hace patente cuando se recuerda que la phronesis, la prudentia, es el concepto central de la filosofía práctica aristotélica y escolástica. Ella es la obra del phroninos y además su más bella obra. Como causa de la separación de la 'prudencia' arguye Hobbes su carácter dependiente del tiempo y del lugar, de las circunstancias, en tanto que mediante un razonamiento correcto se produce nada menos que una "verdad general, eterna e inmutable" (Lev. Cap. 8). La sustitución de una filosofía política dirigida a construir y crear el ámbito del obrar, cobra su plena claridad cuando Hobbes señala las propiedades que deben distinguir al hombre de Estado. Lo que se exige para la organización y mantenimiento (making and maintaining) de un Commonwealth es skill (habilidad), un concepto tomado del mundo de la producción, no de la moral. Este skill consiste en el conocimiento de determinadas reglas iguales a las de la aritmética o de la geometría; no yace sólo en el obrar: "La capacidad para hacer y mantener repúblicas -dice Hobbes- consiste en varias reglas: no en la sola práctica. Construir Estados es cosa diferente a jugar tenis". 


\section{Conclusión:}

Hobbes está convencido de que la crisis del universalismo religioso, el pirronismo en moral, el desorden, la fragmentación y la dispersión -de las sediciones a la tiranía- que resultan particularmente de la guerra civil, dependen de las doctrinas erróneas de escritores antiguos de cosas políticas, para quienes la sociedad perfecta que es el Estado, resulta de una evolución histórica natural y no de la iniciativa racional de individuos que carecen de toda relación hasta que deciden crear el 'estado civil'.

A partir de Hobbes, se puede hablar de un modelo teórico que es adoptado, si bien con variaciones notables, por algunos de los más grandes filosofos políticos de la edad moderna. La preocupación por superar la discordia sin tregua de las opiniones de teólogos, juristas y escritores políticos, así como la seducción producida por la certeza y la armonía que reinaba en el campo de ias disciplinas exactas, son algunas de las razones que llevaron a sostener la tesis de la invariabilidad de supremos ideales del hombre, así como la igualdad eterna y absoluta de su naturaleza.

En Locke, por ejemplo, está presente también la idea de una Etica demostrativa, de tal modo que ". . .de proposiciones evidentes por sí mismas, mediante consecuencias necesarias no menos inconfutables que las de las matemáticas, se podrían derivar las medidas de lo justo y de lo injusto" (. . . ) "Donde no hay propiedad no hay injusticia, es tan cierta como cualquier demostración que se encuentre en Euclides" (Ensayo sobre el entendimiento humano).

Spinoza, retomando el motivo hobbesiano de la política racional, escribe: "Por lo tanto en el dedicarme a la política yo no me he propuesto nada de nuevo y de impensado, sino solamente demostrar con argumentos ciertos e indestructibles, o sea de deducir de la condición misma de la naturaleza humana, los principios que armonizan perfectamente con la práctica. Y para proceder en esta investigación científica con la misma libertad de espíritu con la que acostumbramos aplicarnos a las matemáticas, he hecho un estudio para no reír ni llorar sobre las acciones humanas" (Tratado Político). 
Por su parte, Leibniz respecto de la ciencia del derecho, señala: "La teoría del derecho es de las que no dependen de experimentos, sino de definiciones" (. . .) "se puede comprender que alguna cosa sea justa cuando incluso no haya ninguno que pueda hacerla valer, de igual manera que como sucede en la matemática, donde las relaciones aritméticas son verdaderas, aunque no haya quien enumere ni haya cosas para enumerar" (Elementos del Dereho Natural).

Será el historicismo en sus diversas formas que, permaneciendo en pie contra la crítica racionalista a la autoridad de la historia, condenará en bloque a todos aquellos que, aún perteneciendo a orientaciones metafísicas diferentes, aún enarbolando conclusiones politicas opuestas, igualmente atrafdos por el éxito de las ciencias físicas y por la idea de encontrar un orden racional en el mundo humano, habran emprendido la tentativa de construir un sistema universal que diera cuenta de la praxis, válido para todo tiempo y para todo lugar.

\section{RESUMEN}

El espíritu científico del siglo XVII, inclinado a demostrar la verdad more geométrico y a sostener el ideal de un conocimiento seguro que dé cuenta de la totalidad de lo real partiendo de principios evidentes, influye de manera determinante sobre la justificación racional de las condiciones más adecuadas para la ordenación de la vida en sociedad. En esta línea se inscribe el ambicioso proyecto de Tomas Hobbes, al postular una filosofía práctica demostrativa capaz de articular definitivamente la naturaleza humana, el "estado natural" de la sociedad y el ideal de un orden jurídico-normativo cuya expresión máxima, el Estado como ente de razón, asume la forma de un "cuerpo artificial" construído técnicamente por los hombres. La contrastación con el pensamiento de Aristóteles, permite ver que con Hobbes no sólo nos encontramos frente a una original teoría del poder, sino ante la manifestación naciente de un movimiento cultural, filosófico, científico y político, cuyos efectos de sentido nos son hoy contemporáneos. 